\title{
The antioxidative effects of some medicinal plants as hypoglycemic agents on chromosomal aberration and abnormal nucleic acids metabolism produced by diabetes stress in male adult albino rats
}

\author{
Sherifa H. Salah ${ }^{1}$, Halima S. Abdou ${ }^{1}$, Amal S. Abd El Azeem ${ }^{2}$, Abdel-Rahim E. $^{3}$ \\ ${ }^{1}$ Cell Biology Department, National Research Center, Cairo, Egypt; \\ ${ }^{2}$ Food Science and Nutrition Department, National Research Center, Cairo, Egypt; \\ ${ }^{3}$ Department of Biochemistry, Fac. Agric. Cairo. University.
}

Received 15 January 2011; revised 14 February 2011; accepted 21 February 2011.

\section{ABSTRACT}

The objectives of the present study are to evaluate the chromosomal aberrations in somatic and germ cells of alloxanized diabetic rats treated with methanolic extracts of Cleome droserifolia (Sammo), Anastatica hierochuntica (Kafta), Juniperus phoencea (Araar) and $\mathrm{Hy}$ phaene thebaica (Doum) as well as their effects on blood glucose, liver and testes total soluble protein, DNA and RNA contents and also nucleases (RNAase and DNAase) activity. The material and Methods:The study was performed in six groups. The first group was the normal healthy control; the second group was rats injected with alloxan (diabetic control). Every one of the other four groups (diabetic rats) was ingested with each of the methanolic extracts of Kafta, Doum, Sammo and Araar (individually). The results: The results of chromosomal analysis showed that, diabetic rats had a highly significant increase of chromosomal aberrations compared to that of normal healthy control. Animals which treated with the four plants methanolic extracts showed significant improvements in the frequencies of chromosomal aberrations. In case of biochemical analysis, blood glucose level was significantly increased but immunoglobulins levels were decreased in diabetic albino rats. Diabetes increased serum total lipids, cholesterol, triglycerides and LDL but serum HDL was decreased. RNA and DNA as well as inhibited the nucleases (RNAase and DNAase) activity of both organs (liver and testes) tissue. The conclusions: It is concluded that diabetes is much harmful in the animal body, whilst the induction of flavonoids extract (by methanol) of Kafta, Somma, Araar and Doum reduced these harmful of diabetes.

Keywords: Alloxan: Recrystallized, Methanolic Extracts of Anastatica Hierochuntica (Kafta) Hyphaene Thebaica (Doum) Cleome Droserfolia (Sammo) Juniperus Phoenicea (Araar) and Rattus Norvegicus

\section{INTRODUCTION}

Diabetes mellitus is a metabolic disease and an organ autoimmune disease, characterized by chronic hyperglycemia and disturbances of animal metabolism associated with insulin deficiency and/or action resulting in long term multi-organ complication [1]. Depending on the severity of the metabolic abnormality diabetes may asymptomatic, or may be associated to symptoms (thirst polyuria and weight loss) or may be progress to ketoacidosis and coma [2]. It is associated with both acute and chronic complecation, chronic hyperglycemia cause damage to the eye, kidneys, liver, nerves, heart and blood vessels [3].

Increasing of side effects and disease caused by chemotherapeutics agents and drugs arouse attention toward natural and natural products drugs to minimize hazards caused by these drugs. Flavonoids are among the compounds that commonly occur in plants and are frequent components of the human diet. The antioxidative characters of these compounds was reported before in several studies $[4,5]$.

Oxidative stress has been implicated in diseases ranging from cancer to cardiovascular disease to diabetes to age-related mascular degeneration (AMD) and even to aging process itself [6]. Diabetes related to oxidative stress has potentially serious consequences and limited 
treatment research has focused on arresting the oxidative damage that may be involved in disease initiation or progression. The American Diabetes Association (ADA) (2001) concluded that any improvement in blood glucose level control slows development/ progression of microvascular complication.

There is not available data was observed in literature about the items of chromosomal aberration and nucleic acids system as well as immuno system and lipid profile in diabetic animals which treated with medicinal plants or their extracts. However, the present studies throw light on the diabetes effect on liver, protein and nucleic acids content as well as the activity of nucleases which are in relation with nucleic acids and also serum immunoglubulins (IgG, IgA and $\operatorname{IgM}$ ) and lipid profile.

Experiments were done to elucidate the effect of inclusion of four Egyptian medicinal plants (as antidiabetic agents) source rich in flavonoids content (methanolic extract) on the chromosomal aberration and nucleic acids system as well as immunosystem and profile lipid in alloxanized diabetic adult male albino rats.

\section{MATERIALS AND METHODS}

\subsection{Chemicals}

Alloxan: recrystallized, used to produce insulin - dependent diabetes experimentally.

\subsection{Plant Matrials}

Methanolic extracts of Anastatica hierochuntica (Kafta) Hyphaene thebaica (Doum) Cleome droserfolia (Sammo) Juniperus phoenicea (Araar) are used as antidiabetic plants.

\subsection{Animals}

Adult male rats (36 rats) (Rattus norvegicus) weighted 100-120 gm obtained from a close random bread colony at National Research Center. The animals had free access to standard pelleted diet and tap water. Standard diet consists of casein $15 \%$, cotton seed oil $10 \%$, salt mixture $4 \%$, vitamins mixture $1 \%$, starch $65 \%$ and cellulose $5 \%$ [7].

\subsection{Experimental Design}

Adult male rats were divided equally into six groups:

1) Normal group (control healthy group) received only distilled water.

2) Alloxan injected group: (Group 2) animals were injected with alloxan (60 mg/kg b.wt. interperitoneally) to produce insulin-dependent diabetes experimentally (diabetic control) [8].

3) Diabetic rats treated with plants extracts: Animals of these groups $(3,4,5$ and 6) were administered with
$150 \mathrm{mg} / \mathrm{kg}$ b.w. of methanolic extract of Kafta, Doum, Sammo and Araar for 15 consecutive days [9].

\subsection{Chromosomal Analysis}

The male rats were sacrificed $24 \mathrm{~h}$. after the last treatment.

The first test: chromosomes of bone marrow (somatic cells) were prepared according to the method adapted by Yosida and Amano [10] with slight modifications.

The second test: chromosomes of spermatogonial cells (stem cells of sperms) were prepared according to Brewen and Preston [11].

Spermatocytes were also prepared according to the method adapted by Evans et al., [12] indicating the aberration in the germ cells.

Slides were prepared and 50 metaphases were studied for each animal scoring the different types of chromosomal aberrations in bone marrow, spermatogonia and spermatocytes of rats. Numerical aberrations were also studied and recorded.

The mitotic activity of somatic and germ cells (spermatogonial cells) were determined for treated and control animals. It is expressed by the mitotic index (MI: number of dividing cells in 10000 cells / group).

\subsection{Biochemical Studies}

At the end of the experimental period, animals were killed by decapitation. Blood, liver and testes of the experimental animals were collected. Liver and testes chilled up for analysis and blood serum was prepared by centrifugation at $3000 \mathrm{rpm}$ and chilled up for analysis.

Determination of blood glucose was adopted using the method of Trinder [13] RNA and DNA were determined in liver and testes tissue according to the procedure described by [14] and Schneider [15]. Total soluble protein of both organs tissue was carried out according to Lawary et al [16] method. DNAase and RNAase activities of liver and testes tissues were dete rmined according to the methods described byBergmeyer [17].

\subsection{Statistical Analysis}

Data for chromosomal analysis were analyzed using Chi-square test [18]. A statistical significance between the treated and control groups was done.

Results of the biochemical; studies were analyzed to calculate the statistical significance between the treated and control groups using the statistical package for social science [19] program version 10.

\section{RESULTS}

\subsection{As regards to Cytogenetic Effects}




\subsubsection{Somatic Cells (Bone Marrow Cells)}

The chromosomal examination in this study showed that the structural aberrations include gaps, breaks, fragments, deletions and endomitosis. Numerical aberrations involved polyploidy, hyperploidy (included cells with 41 and 43 chromosomes) and hypoploidy (included 38 and 39 chromosomes) were also studied and showed differences from that of the normal control group (Table 1), in the four tested plants and Alloxan group (diabetic rats).

Total chromosomal aberrations showed highly significant increase $(P<0.001)$ in diabetic rats compared to the normal group. Treatment with Kafta, Doum, Sammo and Araar inhibited the number of chromosomal aberrations in diabetic rats. This inhibition was found to be significant when compared to untreated diabetic animals, but it remains significantly higher than the normal control group.

The main frequent types of structural aberrations were deletions, endomitosis and gaps, which showed a significant increase $(\mathrm{p}<0.001, \mathrm{p}<0.01, \mathrm{pp}<0.05)$. The mitotic index showed a highly significant depression after injection with Alloxan (in diabetic rats) which increase near to the control after treatment with the methanolic extracts of the four plants used.

\subsubsection{Spermatogonia}

The chromosomal examination of spermatogonial cells in diabetic animals after and before treatment with plants showed more frequencies of structural and numerical aberrations of chromosomes than the normal group (Table 2). The diabetic rats had the most frequent of chromosomal aberrations (structural and numerical, $\mathrm{p}$ $<0.001$ ) when compared to the other groups. Treatment with the methanolic extracts of the four plants inhibited the induced chromosomal aberrations significantly. The main frequent types of structural aberrations were deletions and endomitosis.

The number of dividing cells (MI) decreased significantly in diabetic animals and showed a significant increase after treatment with the medicinal plants used in the study.

\subsubsection{Spermatocytes}

Table 3 presents the results of structural and numerical abnormalities in spermatocytes of all studied groups. The observed types of abnormalities were $\mathrm{x}$-y univalent, autosomal univalent, rings and chain. Diabetic rats (Alloxanized) showed a highly significant increase at level $(p<0.001)$ for total structural aberrations compared to the normal group (control). At all types of aberrations, the treatment with medicinal plants inhibited abnormalities significantly but not normalized it. Numerical aberrations were recorded (Table 3 ) and showed a highly significant increase $(P<0.001)$ in diabetic rats, which decreased significantly after treatment with the extracts of medicinal plants.

Cytogenetic studies showed that Araar and Doum had the lowest percentages of total structural aberrations, but generally statistical analysis showed that there were no significant differences between the four medicinal plants used.

Table 1. Mean percentages $\pm \mathrm{SE}$ of chromosomal aberrations in rat bone marrow cells after treatment with Kafta, Doum, Sammo and Araar.

\begin{tabular}{|c|c|c|c|c|c|c|c|c|c|c|c|c|c|}
\hline & \multirow[b]{2}{*}{ Treatment } & \multicolumn{6}{|c|}{ Structural aberrations } & \multirow{2}{*}{$\begin{array}{c}\text { Total } \\
\text { structural } \\
\text { aberr. S }\end{array}$} & \multicolumn{3}{|c|}{ Numerical aberr. S } & \multirow{2}{*}{$\begin{array}{c}\text { Total nu- } \\
\text { merical } \\
\text { aberra. S }\end{array}$} & \multirow{2}{*}{$\begin{array}{c}\% \text { of } \\
\text { mitotic } \\
\text { activities }\end{array}$} \\
\hline & & $\begin{array}{l}\text { Chromatid } \\
\text { gaps }\end{array}$ & $\begin{array}{c}\text { Chromosmal } \\
\text { gaps }\end{array}$ & $\begin{array}{c}\text { Chromatid } \\
\text { breaks }\end{array}$ & Fragments & Deletions & Endomitosis & & Hypoploidy & Hyperploidy & Polyploidy & & \\
\hline \multirow{5}{*}{$\begin{array}{c}\text { Mean } \pm \\
\text { S.E. }\end{array}$} & 1-Control & $\begin{array}{c}0.8 \\
\pm \\
0.4090\end{array}$ & $\begin{array}{c}0.0 \\
\pm \\
0.0\end{array}$ & $\begin{array}{c}0.0 \\
\pm \\
0.0\end{array}$ & $\begin{array}{c}0.0 \\
\pm \\
0.0\end{array}$ & $\begin{array}{c}0.4 \\
\pm \\
0.3309\end{array}$ & $\begin{array}{c}0.0 \\
\pm \\
0.0\end{array}$ & $\begin{array}{c}1.2 \\
\pm \\
0.5106\end{array}$ & $\begin{array}{c}1.0 \\
\pm \\
0.4472\end{array}$ & $\begin{array}{c}0.0 \\
\pm \\
0.0\end{array}$ & $\begin{array}{c}0.0 \\
\pm \\
0.0\end{array}$ & $\begin{array}{c}1.0 \\
\pm \\
0.4472\end{array}$ & 6.3 \\
\hline & 2-Alloxan & $\begin{array}{l}3.0 \pm * \\
0.3760\end{array}$ & $\begin{array}{c}1.8 \\
\pm \\
* * 0.4090\end{array}$ & $\begin{array}{c}0.6 \\
\pm \\
0.3309\end{array}$ & $\begin{array}{c}0.6 \\
\pm \\
0.3309\end{array}$ & $\begin{array}{c}3.0 \\
\pm * * \\
0.3760\end{array}$ & $\begin{array}{c}2.4 \\
\pm * * * \\
0.3309\end{array}$ & $\begin{array}{c}11.4 \\
\pm * * * \\
0.5785\end{array}$ & $\begin{array}{c}2.6 \\
\pm * \\
0.4229\end{array}$ & $\begin{array}{c}1.0 \\
\pm * \\
0.3760\end{array}$ & $\begin{array}{c}1.8 \\
\pm * * \\
0.4090\end{array}$ & $\begin{array}{c}5.4 \\
\pm * * * \\
0.6243\end{array}$ & 3.4 \\
\hline & $\begin{array}{l}\text { 3-Alloxan } \\
+ \text { Kafta }\end{array}$ & $\begin{array}{c}2.4 \\
\pm * 0.4229\end{array}$ & $\begin{array}{c}0.6 \\
\pm \\
0.4229\end{array}$ & $\begin{array}{c}0.8 \\
\pm * \\
0.2990\end{array}$ & $\begin{array}{c}0.4 \\
\pm \\
0.3309\end{array}$ & $\begin{array}{c}2.2 \\
\pm * \\
0.2990\end{array}$ & $\begin{array}{c}1.8 \\
\pm * * \\
0.2990\end{array}$ & $\begin{array}{c}8.2 \\
\pm * * * \\
0.5106\end{array}$ & $\begin{array}{c}2.2 \\
\pm \\
0.2990\end{array}$ & $\begin{array}{c}1.2 \\
\pm * \\
0.2990\end{array}$ & $\begin{array}{c}1.0 \\
\pm * \\
0.4472\end{array}$ & $\begin{array}{c}4.4 \\
\pm * * * \\
0.3309\end{array}$ & 5.1 \\
\hline & $\begin{array}{l}\text { 4-Alloxan } \\
+ \text { Doum }\end{array}$ & $\begin{array}{c}1.6 \\
\pm \\
0.4229\end{array}$ & $\begin{array}{c}0.6 \\
\pm \\
0.3309\end{array}$ & $\begin{array}{c}0.4 \\
\pm \\
0.3309\end{array}$ & $\begin{array}{c}0.2 \\
\pm \\
0.2990\end{array}$ & $\begin{array}{c}1.8 \\
\pm * \\
0.2990\end{array}$ & $\begin{array}{c}1.0 \\
\pm * \\
0.3760\end{array}$ & $\begin{array}{c}5.6 \\
\pm * * * \\
0.4229\end{array}$ & $\begin{array}{c}1.4 \\
\pm \\
0.3309\end{array}$ & $\begin{array}{c}0.4 \\
\pm \\
0.3309\end{array}$ & $\begin{array}{c}0.6 \\
\pm \\
0.3309\end{array}$ & $\begin{array}{c}2.4 \\
\pm \\
0.4775\end{array}$ & 5.1 \\
\hline & $\begin{array}{l}\text { 6-Alloxan } \\
+ \text { Araar }\end{array}$ & $\begin{array}{c}2.2 \\
\pm \\
0.409\end{array}$ & $\begin{array}{c}0.4 \\
\pm \\
0.3309\end{array}$ & $\begin{array}{c}0.2 \\
\pm \\
0.2990\end{array}$ & $\begin{array}{c}0.4 \\
\pm \\
0.3309\end{array}$ & $\begin{array}{c}1.4 \\
\pm \\
0.3309\end{array}$ & $\begin{array}{c}0.6 \\
\pm \\
0.3309\end{array}$ & $\begin{array}{c}5.2 \\
\pm * * * \\
0.5106\end{array}$ & $\begin{array}{c}0.8 \\
\pm \\
0.2990\end{array}$ & $\begin{array}{c}0.4 \\
\pm \\
0.3309\end{array}$ & $\begin{array}{c}1.0 \\
\pm * \\
0.4472\end{array}$ & $\begin{array}{c}2.2 \\
\pm \\
0.5106\end{array}$ & 5.1 \\
\hline
\end{tabular}

* Significant at $\mathrm{P}<0.05$, ** at $\mathrm{P}<0.01$ and $* * *$ at $\mathrm{P}<0.001$. 
Table 2. Mean percentages $\pm \mathrm{SE}$ of chromosomal aberrations in rat spermatogonial cells after treatment with Kafta, Doum, Sammo and Araar.

\begin{tabular}{|c|c|c|c|c|c|c|c|c|c|c|c|c|}
\hline \multirow{2}{*}{ Treatment } & \multicolumn{6}{|c|}{ Structural aberrations } & \multirow{2}{*}{$\begin{array}{c}\text { Total } \\
\text { structural } \\
\text { abber.S }\end{array}$} & \multicolumn{3}{|c|}{ Numerical aberr. S. } & \multirow{2}{*}{$\begin{array}{l}\text { Total numerical } \\
\text { aberra. S }\end{array}$} & \multirow{2}{*}{$\begin{array}{c}\% \text { of mitotic } \\
\text { activities }\end{array}$} \\
\hline & $\begin{array}{c}\text { Chromatid } \\
\text { gaps }\end{array}$ & $\begin{array}{c}\text { Chromosmal } \\
\text { gaps }\end{array}$ & $\begin{array}{c}\text { Chromatid } \\
\text { breaks }\end{array}$ & Fragments & Deletions & s Endomitosis & & Hypoploidy & Hyperploidy & Polyploidy & & \\
\hline 1-Control & $\begin{array}{c}0.8 \\
\pm \\
0.299\end{array}$ & $\begin{array}{c}0.0 \\
\pm \\
0.0\end{array}$ & $\begin{array}{c}0.0 \\
\pm \\
0.0\end{array}$ & $\begin{array}{c}0.0 \\
\pm \\
0.0\end{array}$ & $\begin{array}{c}0.2 \\
\pm \\
0.299\end{array}$ & $\begin{array}{c}0.0 \\
\pm \\
0.0\end{array}$ & $\begin{array}{c}0.8 \\
\pm \\
0.299\end{array}$ & $\begin{array}{c}0.2 \\
\pm \\
0.29\end{array}$ & $\begin{array}{c}0.0 \\
\pm \\
0.0\end{array}$ & $\begin{array}{c}0.2 \\
\pm \\
0.29\end{array}$ & $\begin{array}{c}0.4 \\
\pm \\
0.33\end{array}$ & 4.3 \\
\hline 2-Alloxan & $\begin{array}{l}2.6 \\
\pm * \\
0.42\end{array}$ & $\begin{array}{c}0.2 \\
\pm \\
0.299\end{array}$ & $\begin{array}{c}1.0 \\
\pm * \\
0.38\end{array}$ & $\begin{array}{c}0.8 \\
\pm * \\
0.299\end{array}$ & $\begin{array}{c}2.8 \\
\pm * * * \\
0.41\end{array}$ & $\begin{array}{c}2.2 \\
\pm * * * \\
0.41\end{array}$ & $\begin{array}{c}9.6 \\
\pm * * * \\
0.423\end{array}$ & $\begin{array}{l}1.8 \\
\pm * \\
0.41\end{array}$ & $\begin{array}{c}0.6 \\
\pm \\
0.331\end{array}$ & $\begin{array}{l}1.6 \\
\pm \quad * \\
0.331\end{array}$ & $\begin{array}{c}4.0 \\
\pm \quad * * * \\
0.447\end{array}$ & 2.8 \\
\hline $\begin{array}{l}\text { 3-Alloxan + } \\
\text { Kafta }\end{array}$ & $\begin{array}{c}1.2 \\
\pm \\
0.4090\end{array}$ & $\begin{array}{c}0.0 \\
\pm \\
0.0\end{array}$ & $\begin{array}{c}0.4 \\
\pm \\
0.3309\end{array}$ & $\begin{array}{c}0.2 \\
\pm \\
0.2990\end{array}$ & $\begin{array}{c}1.2 \\
\pm \\
0.2990\end{array}$ & $\begin{array}{c}1.0 \\
\pm * \\
0.3760\end{array}$ & $\begin{array}{c}4.0 \\
\pm * * * \\
0.4472\end{array}$ & $\begin{array}{c}0.6 \\
\pm \\
0.3309\end{array}$ & $\begin{array}{c}0.0 \\
\pm \\
0.0\end{array}$ & $\begin{array}{c}1.0 \\
\pm \\
0.4472\end{array}$ & $\begin{array}{c}1.6 \\
\pm \\
0.4229\end{array}$ & 3.9 \\
\hline $\begin{array}{l}\text { 4-Alloxan + } \\
\text { Doum }\end{array}$ & $\begin{array}{c}1.8 \\
\pm \\
0.4090\end{array}$ & $\begin{array}{c}0.2 \\
\pm \\
0.2990\end{array}$ & $\begin{array}{c}0.2 \\
\pm \\
0.2990\end{array}$ & $\begin{array}{c}0.0 \\
\pm \\
0.0\end{array}$ & $\begin{array}{c}1.2 \\
\pm \\
0.4090\end{array}$ & $\begin{array}{c}1.0 \\
\pm \quad * \\
0.3760\end{array}$ & $\begin{array}{c}4.4 \\
\pm * * * \\
0.4229\end{array}$ & $\begin{array}{c}1.2 \\
\pm \\
0.2990\end{array}$ & $\begin{array}{c}0.0 \\
\pm \\
0.0\end{array}$ & $\begin{array}{c}1.0 \\
\pm \\
0.4472\end{array}$ & $\begin{array}{c}2.2 \\
\pm * \\
0.5106\end{array}$ & 4 \\
\hline $\begin{array}{l}\text { 5-Alloxan + } \\
\text { Sammo }\end{array}$ & $\begin{array}{c}1.8 \\
\pm \\
0.4090\end{array}$ & $\begin{array}{c}0.0 \\
\pm \\
0.0\end{array}$ & $\begin{array}{c}0.2 \\
\pm \\
0.2990\end{array}$ & $\begin{array}{c}0.0 \\
\pm \\
0.0\end{array}$ & $\begin{array}{c}1.6 \\
\pm * \\
0.3309\end{array}$ & $\begin{array}{c}0.8 \\
\pm * \\
0.2990\end{array}$ & $\begin{array}{c}4.4 \\
\pm * * * \\
0.3309\end{array}$ & $\begin{array}{c}1.0 \\
\pm \\
0.3760\end{array}$ & $\begin{array}{c}0.6 \\
\pm \\
0.3309\end{array}$ & $\begin{array}{c}0.8 \\
\pm \\
0.4090\end{array}$ & $\begin{array}{c}2.4 \\
\pm * * \\
0.5180\end{array}$ & 3.8 \\
\hline $\begin{array}{l}\text { 6-Alloxan + } \\
\text { Araar }\end{array}$ & $\begin{array}{c}1.6 \\
\pm \\
0.3309\end{array}$ & $\begin{array}{c}0.8 \\
\pm \\
* 0.2990\end{array}$ & $\begin{array}{l}0.8 \pm * \\
0.2990\end{array}$ & $\begin{array}{c}0.0 \\
\pm \\
0.0\end{array}$ & $\begin{array}{c}1.6 \pm * * \\
0.3309\end{array}$ & $\begin{array}{l}1.2 \pm * \\
0.2990\end{array}$ & $\begin{array}{c}6.0 \pm \\
* * * \\
0.3760\end{array}$ & $\begin{array}{l}1.4 \pm * \\
0.3309\end{array}$ & $\begin{array}{c}0.6 \\
\pm \\
0.3309\end{array}$ & $\begin{array}{c}1.2 \pm \\
0.46809\end{array}$ & $\begin{array}{c}3.2 \\
\pm * * * \\
0.5981\end{array}$ & 3.5 \\
\hline
\end{tabular}

* Significant at $\mathrm{P}<0.05$, ** at $\mathrm{P}<0.01$ and $* * *$ at $\mathrm{P}<0.001$.

Table 3. Mean percentages of chromosomal aberrations in rat spermatocytes after treatment with Kafta, Doum, Sammo and Araar.

\begin{tabular}{|c|c|c|c|c|c|c|c|c|c|c|c|c|c|c|c|c|c|c|c|}
\hline Group & $\begin{array}{c}\text { Number } \\
\text { of } \\
\text { exam- } \\
\text { ined cells }\end{array}$ & \multicolumn{2}{|c|}{$\begin{array}{l}\text { X-Y univa- } \\
\text { lents }\end{array}$} & \multicolumn{2}{|c|}{ Chain } & \multicolumn{2}{|c|}{ Ring } & \multicolumn{2}{|c|}{$\begin{array}{l}\text { Autosomal } \\
\text { univalents }\end{array}$} & \multicolumn{2}{|c|}{ Total } & \multicolumn{2}{|c|}{ Polyploidy } & \multicolumn{2}{|c|}{ N-1 } & \multicolumn{2}{|c|}{$N+1$} & \multicolumn{2}{|c|}{ Total } \\
\hline $\begin{array}{l}1 . \\
\text { Control }\end{array}$ & 250 & 1 & 0.4 & 0 & 0.0 & 0 & 0.0 & 1 & 0.4 & 2 & 0.8 & 1 & 0.4 & 0 & 0.0 & 0 & 0.0 & 1 & 0.4 \\
\hline $\begin{array}{l}2 . \\
\text { Alloxan }\end{array}$ & 250 & 23 & $9.2 * * *$ & 18 & $7.2 * * *$ & 10 & $4.0 * *$ & 18 & $7.2 * * *$ & 69 & $27.6^{* * *}$ & 15 & $6.0 * * *$ & 9 & $3.6^{* *}$ & 5 & $2.0^{*}$ & 29 & $11.6^{* * *}$ \\
\hline $\begin{array}{l}3 . \\
\text { Alloxan } \\
+ \text { Kafta }\end{array}$ & 250 & 15 & 6.0 & 10 & $4.0^{* *}$ & 5 & $2.0^{*}$ & 10 & $4.0 * *$ & 40 & $16.0 * * *$ & 8 & $3.2 *$ & 2 & 0.8 & 2 & 0.4 & 12 & $4.8 * * *$ \\
\hline $\begin{array}{l}4 . \\
\text { Alloxan } \\
+ \text { Doum }\end{array}$ & 250 & 13 & $5.2^{* *}$ & 9 & $3.6^{* *}$ & 4 & $1.6^{*}$ & 8 & $3.2^{*}$ & 34 & $13.6^{* * *}$ & 5 & 2.0 & 2 & 0.8 & 1 & 0.4 & 8 & $3.2 *$ \\
\hline $\begin{array}{l}5 . \\
\text { Alloxan } \\
+ \text { Sammo }\end{array}$ & 250 & 15 & $6.0 * * *$ & 12 & $4.8 * * *$ & 3 & 1.2 & 9 & $3.6^{* *}$ & 39 & $15.6^{* * *}$ & 6 & 2.4 & 3 & 1.2 & 2 & 0.8 & 11 & $4.4 * *$ \\
\hline
\end{tabular}

* Significant at $\mathrm{P}<0.05, * *$ at $\mathrm{P}<0.01$ and $* * *$ at $\mathrm{P}<0.001$.

\subsection{Biochemical Effects and Immunosystem}

In connection of the present biochemical analysis of liver and testes tissues to evaluate the four plants methanolic extracts, diabetes caused in animals and aberrations in blood glucose metabolism which inhibited the utilization of glucose in the body tissues. Table 4 showed that diabetes produced highly significant increases in blood glucose content $(428 \%$ at normal control), but the four methanolic extracts of the medicinal studied plants ingestion (individually) highly significant reduced the elevated blood glucose in diabetic rats and the reduced values were around that of normal control.
In addition, total soluble protein of liver and testes results of the experimental rats were presented in Table 4. These results showed that diabetes significantly decreased total soluble protein to $67 \%$ and $73 \%$ at normal control, respectively for liver and testes. The ingestion of methanolic extracts of the present studied plants improved these abnormal contents which were $101-102 \%$ and $107-110 \%$ at normal control for liver and testes tissues, respectively of diabetic rats.

As regards to nucleic acids system of liver and testes, the contents of DNA and RNA (as shown in Table 5) were decreased by diabetes to $51 \%$ and $60 \%$ for DNA 
Table 4. Influences of Kafta, Doum, Somma and Araar methanolic extracts on blood glucose and total soluble protein of liver and testes tissues of diabetic male adult albino rats.

\begin{tabular}{|c|c|c|c|c|c|c|}
\hline \multirow{2}{*}{ Treatments } & \multicolumn{4}{|c|}{ Total soluble protein (mg / g tissues) } & \multicolumn{2}{|c|}{ Blood glucose } \\
\hline & Liver (mean \pm SD) & $\%$ & Tests (mean \pm SD) & $\%$ & $\mathrm{mg} / \mathrm{dl}$ & $\%$ \\
\hline Normal control & $186.60^{\mathrm{a}} \pm 4.11$ & 100 & $101.70^{\mathrm{a}} \pm 2.17$ & & $77.60^{\mathrm{a}} \pm 1.51$ & 100 \\
\hline Diabetic control & $124.20^{\mathrm{b}} \pm 3.23$ & 67 & $74.50^{\mathrm{b}} \pm 1.04$ & 73 & $339.60^{\mathrm{b}} \pm 11.20$ & 428 \\
\hline Kafta & $188.80^{\mathrm{a}} \pm 4.02$ & 101 & $109.10^{\mathrm{a}} \pm 2.84$ & 107 & $81.40^{\mathrm{a}} \pm 1.61$ & 105 \\
\hline Doum & $189.00^{\mathrm{a}} \pm 4.44$ & 101 & $112.10^{\mathrm{a}} \pm 3.24$ & 110 & $82.20^{\mathrm{a}} \pm 1.56$ & 106 \\
\hline Sammo & $190.0^{\mathrm{a}} \pm 4.87$ & 102 & $111.0^{\mathrm{a}} \pm 3.02$ & 109 & $83.4^{\mathrm{a}} \pm 1.99$ & 107 \\
\hline Araar & $190.1^{\mathrm{a}} \pm 5.12$ & 102 & $110.8^{\mathrm{a}} \pm 2.99$ & 109 & $84.2^{\mathrm{a}} \pm 1.39$ & 109 \\
\hline LSD 5\% & 21.11 & & 11.41 & & 9.21 & \\
\hline
\end{tabular}

$\%$ relative to normal; Values are significantly different at normal control $(\mathrm{p}<0.05)$.

Table 5. Influences of Kafta, Doum, Somma and Araar methanolic extracts on DNA and RNA contents of liver and testes tissues of diabetic male adult albino rats.

\begin{tabular}{|c|c|c|c|c|c|c|c|c|}
\hline \multirow{2}{*}{ Treatments } & \multicolumn{4}{|c|}{ Liver (mean \pm SD) } & \multicolumn{4}{|c|}{ Tests (mean \pm SD) } \\
\hline & DNA mg/g & $\%$ & RNA mg/g & $\%$ & DNA mg/g & $\%$ & RNA mg/g & $\%$ \\
\hline Normal control & $0.412^{\mathrm{a}} \pm 0.012$ & 100 & $0.208^{a} \pm 0.007$ & 100 & $0.315^{\mathrm{a}} \pm 0.003$ & 100 & $0.182^{a} \pm 0.005$ & 100 \\
\hline Diabetic control & $0.212^{b} \pm 0.004$ & 51 & $0.144^{b} \pm 0.003$ & 69 & $0.190^{c} \pm 0.014$ & 60 & $0.138^{\mathrm{c}} \pm 0.004$ & 73 \\
\hline Kafta & $0.437^{\mathrm{a}} \pm 0.004$ & 106 & $0.211^{\mathrm{a}} \pm 0.05$ & 101 & $0.358^{\mathrm{a}} \pm 0.007$ & 114 & $0.197^{\mathrm{ab}} \pm 0.003$ & 108 \\
\hline Doum & $0.432^{\mathrm{a}} \pm 0.002$ & 105 & $0.212^{\mathrm{a}} \pm 0.004$ & 102 & $0.359^{\mathrm{a}} \pm 0.010$ & 114 & $0.205^{\mathrm{b}} \pm 0.002$ & 113 \\
\hline Sammo & $0.436^{\mathrm{a}} \pm 0.003$ & 106 & $0.218^{\mathrm{a}} \pm 0.007$ & 105 & $0.367^{\mathrm{ab}} \pm 0.018$ & 117 & $0.202^{b} \pm 0.004$ & 111 \\
\hline Araar & $0.435^{\mathrm{a}} \pm 0.003$ & 106 & $0.219^{\mathrm{a}} \pm 0.004$ & 105 & $0.369^{\mathrm{ab}} \pm 0.005$ & 117 & $0.201^{b} \pm 0.007$ & 110 \\
\hline LSD $5 \%$ & 0.030 & & 0.015 & & 0.050 & & 0.020 & \\
\hline
\end{tabular}

$\%$ relative to normal; Values are significantly different at normal control $(\mathrm{p}<0.05)$

and $69 \%$ and $73 \%$ for RNA content, respectively in liver and testes of diabetic rats relative to those of normal healthy control. In contrast, the administration of methanolic extracts of the four medicinal plants (Flavonoids) increased and improved the reduced values of RNA and DNA of diabetic animals which readjusted to those of normal control either in liver or testes which were 101$117 \%$ at those of normal healthy control rats. Consequently, the activity of RNAase and DNAase (nuclease enzymes related to nucleic acids system) were determined in liver and testes tissue of the experimental animals. The obtained results are shown in Table 6, which observed that significant inhibitions were found in the DNAase and RNAase activity of the both organs tissue of diabetic rats related to healthy control. The inhibited activity by diabetes was ranged between $50 \%$ and $61 \%$ at normal control for the two nucleases activity (DNAase and RNAase). This may be due to the oxidative stress in diabetes coexists with reduction in the antioxidant defense system and subsequently leads to the complications of diabetes [38]. Results of Table 6 clearly showed that significant stimulation were caused in the activity of both nucleases of liver and testes tissues of the diabetic rats by ingestion of the methanolic extracts of Kafta, Somma, Doum and Araar. The activity values were im- proved around those of the normal healthy control in which the DNAase activity was $101-103 \%$ at control in liver and testes tissues of diabetic rats ingested with each of the four methanolic extracts but for RNAase the activity was $102 \%$ in liver and $107-112 \%$ in testes tissues of diabetic rats relative to normal healthy control by the same treatments.

In case of immuno system, the changes in serum immunoglobulins (IgG, IgA and IgM) levels of alloxan diabetic rats treated with the methanolic extracts of the four medicinal plants are shown in Table 7. Data revealed that diabetes reduced the three immunoglobulins of serum, but methanolic extracts ingestion of the four medicinal plants significantly increased the serum levels of IgG, IgA and IgM of diabetic rats. Diabetic rats ingested Sammo extract gave the highest levels of the three immunoglobulins when compared with the other used three medicinal plants.

Table 8 showed the effects of methanolic extracts of the four medicinal plants on lipid profile of diabetic rats serum. The results observed that total lipid, LDL-C and total triglycerides (levels of serum of diabetic rats) amounted about 2 fold, while the total cholesterol of serum diabetic rats content was 1.5 fold than that of normal healthy control but HDL-C level of diabetic rats 
Table 6. Influences of Kafta, Doum, Somma and Araar methanolic extracts on DNAase and RNAase activity of liver and testes tissues of diabetic male adult albino rats.

\begin{tabular}{|c|c|c|c|c|c|c|c|c|}
\hline \multirow[b]{2}{*}{ Treatments } & \multicolumn{4}{|c|}{ Liver (mean \pm SD) } & \multicolumn{4}{|c|}{ Tests(mean \pm SD) } \\
\hline & DNAase IU/g & $\%$ & RNAase IU/g & $\%$ & $\begin{array}{c}\text { DNAase } \pm \\
\text { IU/g }\end{array}$ & $\%$ & RNAase IU/g & $\%$ \\
\hline Normal control & $55.51^{\mathrm{a}} \pm 1.11$ & 100 & $40.12^{\mathrm{a}} \pm 0.84$ & 100 & $80.81^{\mathrm{a}} \pm 1.21$ & 100 & $41.11^{\mathrm{a}} \pm 0.81$ & 100 \\
\hline Diabetic control & $33.86^{\mathrm{b}} \pm 0.87$ & 61 & $20.12^{\mathrm{b}} \pm 0.56$ & 50 & $47.77^{\mathrm{b}} \pm 0.97$ & 59 & $21.00^{\mathrm{b}} \pm 0.52$ & 51 \\
\hline Kafta & $55.92^{\mathrm{a}} \pm 1.01$ & 101 & $41.00^{\mathrm{a}} \pm 1.00$ & 102 & $81.27^{\mathrm{a}} \pm 1.78$ & 101 & $44.44^{\mathrm{a}} \pm 0.94$ & 108 \\
\hline Doum & $56.01^{\mathrm{a}} \pm 0.98$ & 101 & $41.08^{\mathrm{a}} \pm 0.87$ & 102 & $81.42^{\mathrm{a}} \pm 2.01$ & 101 & $43.97^{\mathrm{a}} \pm 0.78$ & 107 \\
\hline Sammo & $56.11^{\mathrm{a}} \pm 1.21$ & 101 & $40.92^{\mathrm{a}} \pm 0.79$ & 102 & $83.00^{\mathrm{a}} \pm 1.97$ & 103 & $45.02^{\mathrm{a}} \pm 0.87$ & 110 \\
\hline Araar & $56.10^{\mathrm{a}} \pm 0.94$ & 101 & $41.10^{\mathrm{a}} \pm 0.91$ & 102 & $82.22^{\mathrm{a}} \pm 2.00$ & 102 & $46.01^{\mathrm{a}} \pm 1.00$ & 112 \\
\hline LSD 5\% & 8.11 & & 7.72 & & 15.02 & & 7.04 & \\
\hline
\end{tabular}

$\%$ relative to normal; Values are significantly different at normal control $(\mathrm{p}<0.05)$

Table 7. Mean values of serum immunoglobulins Ig.G, Ig.A and Ig.M of the diabetic rats treated with the four studied plants (mg/dl).

\begin{tabular}{lccc}
\hline \multicolumn{1}{c}{ Groups } & Ig.G & Ig.A & Ig.M \\
\hline Normal control & $2462^{\mathrm{a}} \pm 121.11$ & $335^{\mathrm{a}} \pm 16.61$ & $227^{\mathrm{a}} \pm 10.12$ \\
Control (diabetic) & $2179.82^{\mathrm{c}} \pm 110.23$ & $195.67^{\mathrm{e}} \pm 10.01$ & $166.15^{\mathrm{d}} \pm 9.02$ \\
Araar & $2306.47^{\mathrm{b}} \pm 114.50$ & $258.28^{\mathrm{c}} \pm 13.02$ & $192.41^{\mathrm{c}} \pm 9.99$ \\
Kafla & $2387.50^{\mathrm{ab}} 114.50$ & $231.95^{\mathrm{d}} \pm 12.00$ & $184.0^{\mathrm{c}} \pm 9.03$ \\
Sammo & $2457.67^{\mathrm{b}} \pm 119.24$ & $318.62^{\mathrm{a}} \pm 16.07$ & $235.93^{\mathrm{a}} \pm 11.11$ \\
Doum & $2307.52^{\mathrm{b}} \pm 119.24$ & $288.22^{\mathrm{b}} \pm 13.33$ & $207.28^{\mathrm{b}} \pm 10.24$ \\
LSDS\% & 94.36 & 24.92 & 13.34 \\
\hline
\end{tabular}

Table 8. Mean values of serum lipid parameters of normal rats (mg/dl).

\begin{tabular}{lccccc}
\hline \multicolumn{1}{c}{ Group } & Total lipid mean & T. Cholest mean & Triglycerid mean & $\begin{array}{c}\text { LDL }- \text { Cholest } \\
\text { mean } \pm \text { ISE }\end{array}$ & HDL - Cholest \\
\hline Control normal rats & $256.2^{\mathrm{a}} \pm 12.1$ & $71.7^{\mathrm{a}} \pm 5.0$ & $70.9^{\mathrm{a}} \pm 10.3$ & $12.5^{\mathrm{a}} \pm 1.61$ & $52.79^{\mathrm{a}} \pm 2.1$ \\
Diabetic control & $453.73^{\mathrm{d}} \pm 29.43$ & $103.30^{\mathrm{c}} \pm 10.00$ & $126.41^{\mathrm{e}} \pm 11.61$ & $21.61^{\mathrm{d}} \pm 1.10$ & $45.90^{\mathrm{b}} \pm 2.1$ \\
Kafla extract & $412.53^{\mathrm{cd}} \pm 30.11$ & $85.74^{\mathrm{b}} \pm 7.42$ & $92.17^{\mathrm{c}} \pm 10.02$ & $18.26^{\mathrm{c}} \pm 1.02$ & $53.38^{\mathrm{a}} \pm 3.41$ \\
Doum extract & $378.94^{\mathrm{c}} \pm 20.46$ & $71.81^{\mathrm{a}} \pm 6.66$ & $88.45^{\mathrm{c}} \pm 8.76$ & $10.76^{\mathrm{b}} \pm 0.99$ & $60.58^{\mathrm{c}} \pm 3.87$ \\
Samma extract & $296.20^{\mathrm{b}} \pm 20.31$ & $74.13^{\mathrm{a}} \pm 6.04$ & $80.00^{\mathrm{b}} \pm 7.32$ & $11.94^{\mathrm{a}} \pm 1.02$ & $66.28^{\mathrm{d}} \pm 4.00$ \\
Araar extract & $443.5^{\mathrm{e}} \pm 27.54$ & $90.76^{\mathrm{b}} \pm 8.91$ & $106.01^{\mathrm{d}} \pm 9.94$ & $20.33^{\mathrm{d}} \pm 1.03$ & $47.92^{\mathrm{b}} \pm 3.02$ \\
LSD 5\% & 50.00 & 10.01 & 7.12 & 1.51 & 4.55 \\
\hline
\end{tabular}

serum was significantly decreased relative to that of normal control. Data in Table 8 found that all the used plants methanolic extracts significantly improved the lipid profile of diabetic rats serum in which total lipids, triglycerides, cholesterol and LDL-C contents in serum of the diabetic rats were significantly decreased and the values of ingested diabetic rats were around that of the healthy normal control, but HDL-C level of serum diabetic rats was significantly increased and their levels were around or more than that of healthy ones. The ingestion of Sammo methanolic extracts into diabetic rats produced the highest effects on lipids profile of diabetic rats serum.

It means that, the methanolic extracts (Flavonoids) of Kafta, Somma, Araar and Doum ingestions treated the oxidative stress of diabetes in adult albino rats and the statistical analysis of the present results showed also that, there is insignificant difference between the four studied medicinal Egyptian plants.

\section{DISCUSSION}

Diabetes mellitus is one of the most common metabolic diseases. In many countries the incidence of diabetes mellitus has reached a high percentage. In a survey many of non-insulin-dependent diabetes was found to resort to the use of medicinal plants to treat their diseases [20]. In fact, phytotherpay has been widely used because of the low cost and the easy availability of medicinal plants. However, the studies on antidiabetic plants used are relatively recent and has begun to evolve in the last few years. Moreover, until recently, there has 
been no extensive documentation of data on antidiabetic plants.

In the present study, single injection of $60 \mathrm{mg} / \mathrm{kg} \mathrm{b}$. wt. Alloxan produce insulin-dependent diabetes animals experimentally. Alloxan induced genetic changes in somatic and germ cells. It reduced the number of dividing cells causing inhibition in the mitotic index than normal. Alloxan and streptozotocin (STZ) can destroy $\beta$-cells causing significant increase in blood glucose level. This increase in circulating glucose level is believed to contribute to $\beta$-cell dysfunction [21]. This effect relates to their structure as a DNA alkylating agents, w.hich leads to necrosis of pancreatic beta cells and thus to a state of insulin-dependent diabetes mellitus [22]. The action of these drugs could also be due to alternation in membrane fatty acid content, which may affect $\mathrm{Na}^{+}, \mathrm{K}^{+}$- ATPase activity, membrane fluidity, and fatty acid content [23].

Animals of Alloxan injected group showed more frequent chromosome aberrations (structural and numerical) compared to the control or normal healthy group. It also showed low percentage of mitotic activity of cells. Oral treatment with methanolic extracts of Kafta, Doum, Sammo and Araar inhibited the frequency of chromosomal aberrations significantly in both somatic and germ cells.

Many authors have explained the antidiabetic effect of plants. They may stimulate insulin secretion from $\beta$-cells and induce regeneration, revitalization and/or hyperplasia of the $\beta$-cells. Moreover, extracts of antidiabetic plants can act by initating the action of insulin by occurrence of active principles "an insulin-like action" and enable cells to achieve better utilization of glucose [9]. Antidiabetic plants can act by supplying $\beta$-cells with the necessary elements $\left(\mathrm{Cu}^{++}, \mathrm{Mg}^{++}, \mathrm{Ca}^{++}\right)$and reduce the action of insulinase, an enzyme that destroys the insulin in the liver.

On the other hand, the results indicated that administration of Kafta, Doum, Sammo and Araar plant extracts improved (but not completely normalized) the diabetogenic action induced by Alloxan. It showed a hypoglycemic effect on blood glucose level in these animals. Such hypoglycemic effect could be through increased serum insulin levels provided by repair / regeneration of the endocrine pancreas [24]. This agrees with the results of Das et al. [25] who mentioned that the potential hypoglycemic nature of the leaf extract of Aegle marmelose improved functional state of pancreatic beta cells and help in regeneration of damaged pancrease [26], also added that the hypoglycemic effect of Biophutum sensitivum leaf extract on diabetic rabbits is possibly due to pancreatic beta cell stimulating action.

It is believed that the presence of flavone glycosidic components in the plant extract of Anastatica hierochuntia, is responsible for reducing the blood glucose level in
STZ-diabetic rats [27].

The present results are in agreement with those of Ismail et al. [5], Halima et al. [28], Peyroux and Sternberg [29] and Wirasathian et al. [30]. They reported that diabetes caused an oxidative stress include stimulation lipid peroxidation and protein glycation as well as inhibition of antioxidative enzymes with alteration in the structure and function of collagen and basement membranes as well as reduced tissue soluble protein. Natural flavonoids such as those of Kafta, Somma, Araar and Doum methanolic extracts have been shown to reduce this oxidative stress in experiemental diabetes [31, 32]. The lowering effect of plant flavonoids on diabetes may be due to the increase in insulin-receptor binding followed by increasing the signal sequences [33] or the antioxidant of these flavonoids. The oxidative stress usually results in stimulated superoxide dismutase activity and inhibited insulin binding to insulin receptor [34]. These effects of diabetes were improved by flavonoids treatment (presented in the methanolic extracts of the studied medicinal plants, [35].

In connection, the present studies of nucleic acids system and cytogenetic studies agreed those of our work [29] in which the induction of methanolic extracts of Kafta, Arrar, Somma and Doum into female and pregnant albino rats improved the stress effects of diabetes on tissue DNA and RNA contents and the ingestion of flavonoids methanolic extracts vigrously increased both nucleic acids content in animal tissues of diabetic rats and traffic over alternate enzymatic pathways which stimulated protein biosynthesis. Also, inhibited effects of diabetes on nucleases (RNAase and DNAase) activity may be due to the oxidative stress in diabetes coexists with reduction in the antioxidant defense system and subsequently leads to the complication of diabetes [36]. The improved effects of methanolic extracts on diabetic rats are in agreement with those of Halima - Abdou et al., [29] who found that in pregnant rats, flavonoids extracts improved the injurious effects of diabetes on the activities of nucleases. The stimulation of nucleases activity of diabetic rat tissues under the treatments of flavonoids of plant methanolic extracts may be due to that diabetes caused in animal an oxidative stress which produced great chromosomal aberrations. These damaged DNA and blocked the action of RNA polymerase and so prevent replication process. The resulting damage must be repaired under the induction of flavonoid treatment if DNA is return to its genetic integrity. The DNA damage is excised by the sequential of nuclease leaving a short gap which is filled in by action of polymerase. The final join is affected by polynucleotides ligase [37].

The results in Table 7 of immuno system showed that methanolic extracts of the four medicinal plants used in 
the present experiment improved serum contents of $\mathrm{IgG}$, $\operatorname{IgA}$ and $\operatorname{IgM}$. These results are supported with those obtained by Nahla and Adbel-Nabi[38], they stated that ethanolic extract of Cleome droserifolia significantly increased serum total protein, albumin, $\alpha$-globulin, $\beta$-globulin and $\gamma$-globulin of diabetic rats. These increases may be an expression of the formation of more antibodies, since it is well known that most antibodies generated against particular antigen are mainly lie in $\gamma$-globulin fraction of serum proteins and circulate to lesser extent to $\beta$-globulin area [39]. Further more some of the antibodies have electrophoretic mobilities between $\beta$ and $\gamma$ globulin [40].

Data in Table 8 of lipids of profile observed that the methanolic extracts of the used medicinal plants improved serum lipids profile of diabetic rats in which total lipids, cholesterol, triglycerides and LDL-C levels were reduced but HDL-C was increased in serum of diabetic rats. These results are in agreement with those of Ismail et al.[5] who found the diabetes increased all lipids profile except HDL-C was decreased in rats serum. These disturbed effects were improved by injection with anthocyanin and epicatechin (flavonoid compounds). They found that the administration of flavonoids extracts into diabetic rats significantly increased adiponectin levels that stimulate the hypoglycemic action of insulin without altering the concentration of insulin in blood. Recent studies have revealed that adiponectin plays important roles in the regulation of lipid metabolism. For example, it increases free fatty acid oxidation in the muscle, enhances hepatic insulin action and inhibits foam cell formation by reducing the accumulation of lipids in human macrophages and prevents atherosclerosis.

The decreased serum insulin level may inhibit adiponectin secretion from adipose tissue but flavonoid induction stimulated adiponectin secretion which stimulated the action of insulin in lipid metabolism [5].

The present results are confirmed each other in which the stimulation of DNAase and RNAase activity was paralleled with those of DNA, RNA and protein contents and also with immunoglobulins levels of serum which were increased under the flavonoids ingestion into diabetic animals (observed in the previous results of the present work) and also confirmed by the findings of chromosomal aberrations of the present studies. These may be due to stimulation of protein biosynthesis process to produce the specific factors (included antioxidative enzymes) required to improve and treat the oxidative stress of diabetes by replication and transcription needs a lot amount of nucleotides, which obtained in the tissue cells by hydrolysis of DNA and RNA via nucleases [39].

The present studies concluded that diabetes is much harmful in the animal body, whilst the induction of flavonoids extract (by methanol) of Kafta, Somma, Araar and Doum reduced these harmful of diabetes, which may be to the antiglycemic influences of flavonoids of these medicinal plants on the oxidative stress of diabetes via nucleic acids and protein metabolism.

\section{REFERENCES}

[1] Caughron, K.F. and Smith, E.L. (2002) Definition and description of diabetes mellitus. South Medical Journal, 95, 35-49.

[2] Kuzuya, T., Nakagaw, S., Satoh, J., Kanazawa, Y., Iwamoto, Y. and Kobayashi, M., et al. (2002) Report of the committee of the Japan Diabetes Society on the diagnostic criteria of diabetes mellitus. Diabetes Research and Clinical Practice, 55, 65-85.

doi:10.1016/S0168-8227(01)00365-5

[3] Lebvitz, H.E. (2001) Diagnosis, classification and pathogenesis of diabetes mellitus. The Journal of Clinical Psychiatry, 62, 5-9.

[4] Lotito, S.B. and Frei, B. (2004) Relevance of apple polyphenols as antioxidants in human plasma: Contrasting in vitro and in vivo effects. Free Radical Biology and Medicine, 36, 201-211.

[5] Ismail, S.H., Ahmed, S.A., Fayed, S.A. and Van-Breemen, R.B. (2007) Adiponectin, insulin and nitric oxide levels in hyperlipidemia and hypercholesterolemia in addition of streprozotocin-induced diabetes mellitus and normal rats intraperitonially injected with anthocyanin and epicatechin extracts. New Egyptian Journal of Medicine, 36, 135-143.

[6] Van-Lreshout, M., West, C.E. and Van-Breemen, R.B. (2003) Isotope tracer techniques for study the bioavailiability and inefficacy of dietary coroteniods, particulary B. The American. Journal of Clinical Nutrition, 7, 12-28.

[7] Lane-Petter, W. and Pearson, A. (1971) Dietary requirement. In: The laboratory animal principles and practice. Academic press, London and New York, 142.

[8] Lazaro, A. (1949) Alloxan and pathogenesis of diabetes mellitus. Physiological Reviews, 29, 48.

[9] Abdel-Rahim, E.A., Salah, H., Sherifa, H.S., El-Beltagi S. and Fayed, A.S. (2005) Comparative studies on the influences of Juniperus phoenicea and Hyphaena thebaica as hypoglycemic factors in diabetic rats. Journal of $\mathrm{Ge}$ netic Engineering and Biotechnology, 3, 2.

[10] Yosida, T.H. and Amano, K. (1965) Autosomal polymorphism in laboratory bred and wild Norway rats Rattus norvegicus, found in Misima. Chromosoma, 16, 628-667. doi:10.1007/BF00285115

[11] Brewen, G.J. and Preston, R.J. (1978) Analysis of chromosome aberration in mammalian germ cell. Chemical Mutagens, 5, 127-150.

[12] Evans, E.P., Breckon, G.A. and Ford, C.E. (1964) An airdrying method for meiotic preparation from mammalian test. Cytogenetics, 3, 289-298. doi:10.1159/000129818

[13] Trinder P. (1969) Enzymatic determination of glucose in blood serum. Annals of. Clinical Biochemistry, 6, 24.

[14] Dische, Z., Chargaff, E. and Davidoson, J.M. (1955) Color reaction of nucleic acid components. In: The nucleic acids eds. Academic Press, New York, 1, 270-284. 
[15] Schneider, W. C. (1957) Determination of acid in tissues by pentose analysis. In: Method enzymology. Academic Press, New York, 680-384.

[16] Lowary, O.H., Resabrought, N.J., Farrand, A.L. and Randoll, R.J. (1951) Protein measurements with the folin phenol reagent. The Journal of Biological Chemistry, 193, 267.

[17] Bergmeyer, U.H. (1974) Determination of mucleases activity (RNAse and DNAase) In: Method of Enzymatic analysis. 2nd Edition, Acad. Press Inc., New York, San Francisco and London, 447-511.

[18] Statistical package for the social science. (1999) Inc Chicago.

[19] Snedecor, G.W. and Cochran, W.G. (1967) Statistical methods. 6th Edition, Ames, Lowa, the Lowa state University.

[20] Ziyyat, A., Legssyer, A., Mekhfi, H., Dassouli, A., Serhrouchni, M. and Benjelloun, W. (1997) Phytotherpay of hypertension and diabetes in oriental Morocco. Journal of Ethnopharmacology, 58, 45-54. doi:10.1016/S0378-8741(97)00077-9

[21] Hansen, M. (1998) Pathophysiology: Foundations of diseases and clinical intervention. W.B. Saunders Company, Philadelphia, U.S.A., 851-852.

[22] Elsner, M., Guldbakke, B., Tiedge, M., Munday, R. and Lenzen, S. (2000) Relative importance of transport and alkylation for pancreatic beta-cell toxicity of streptozotocin. Diabetologia, 43, 1528-1533. doi:10.1007/s001250051564

[23] Sennoune, S., Gerbi, A., Duran, M.J., Benkoel, L., Pierre, S., Lambert, R., et al. (1999) A quantitative immunocytochemical study of $\mathrm{Na}^{+}, \mathrm{K}^{+}$ATPase in rat hepatocytes after STZ-induced diabetes and determination of blood glucose using an oxidase-peroxidase system: A non carcinogenic chromagen. Am. Clinical Biochemistry, 6, 2430.

[24] Rahmy, T.R. and El-Ridi, M.R. (2002) Action of Anastatica hierochuntica plant extract on Islets of Langerhans in normal and diabetic rats. Egyptian Journal of Biology, 4, 87-94.

[25] Puri, D. and Baral, N. (1998) Hypoglycemic effect of Biophytum sensitivum in the alloxan diabetic rabbits. Indian Journal of Physiology \& Pharmacology, 42, 401406.

[26] Das, A.V., Padayatti, P.S. and Paulose, C.S. (1996) Effect of leaf extract of Aegle marmelose (L.) Correa ex Roxb. on histological and ultrastructural changes in tissues of streptozotocin induced diabetic rats. Indian Journal of Experimental Biology, 34, 341-345.

[27] Puri, D. and Baral, N. (1998) Hypoglycemic effect of Biophytum sensitivum in the alloxan diabetic rabbits. Indian Journal of Physiology \& Pharmacology, 42, 401406.

[28] Khalifa, T.A. (1980) Pharmacognostical study of certain species of Anastatica. Ph.D. Thesis, Cairo University, Egypt.

[29] Halima, S., Abdou, H., Sherifa, S. and Amira, A.R. (2008) Chromosomal aberrations and nucleic acids systems affected by some Egyptian medicinal plants used in treating female pregnant diabetic rats. Journal of Applied Science.

[30] Wirasathian, L., Pengsuparp, T., Suttisri, R., et al., (2006) Inhibitors of aldose reductase and advanced glycation end-products formation from the leaves of Stelechocarpus Cauliflorus. Phytomedicine, Applied Science, 3.

[31] Peyroux, J. and Sternberg, M. (2006) Advanced glycation end-products (AGEs): Pharmacological inhibition in diabetes. Pathological Biology, 54, 405-419. doi:10.1016/j.patbio.2006.07.006

[32] Wirasathian, L., Pengsuparp, T., Suttisri, R., Ueda, H., Moriyasu, M. and Kawanishi, K. (2006) Inhibitors of aldose reductase and advanced glycation end-products formation from the leaves of Stelechocarpus Cauliflorus. Phytomedicine, Avilable online.

[33] Coskun, O., Kanter, M., Korkmaz, A. and Oter, S. (2005) Quercetin, a flavonoid antioxidant, prevents and protects streptozotocin-induced oxidative stress and $\beta$-cell damage in rat pancreas. Pharmacological research, 51, 117123. doi:10.1016/j.phrs.2004.06.002

[34] Anton, S., Melville, L. and Rena, G. (2007) Epigallocatechin gallate (EGCG) mimics insulin action on the transcription factor FOXOLa and elicits cellular responses in the presence and absence of insulin. Cellular signaling, 19, 378-383. doi:10.1016/j.cellsig.2006.07.008

[35] Rao, T.P., Sakaguchi, N., Juneja, L.R., et al. (2005) (Emblica Officinalis Gaertn) extracts reduce oxidative stress in streptozotocin-induced diabetic rats. Journal of $\mathrm{Me}$ dicinal Food, 8, 362-368. doi:10.1089/jmf.2005.8.362

[36] Baraldi, R., Rapparini, F., Facini, O., et al. (2005) Isoprenoid emission and physiological activities of Mediterranean macchia vegetation under field condition. Mediterranean Ecology, 6, 3-9.

[37] Spines, G.A. (1999) The dual role of nitric acid in islets $\beta$-cells. News in Physiological Sciences, 14, 49-54.

[38] Adams, R.L.P., Knowler, J.T. and Leader. D.P. (1993) The biochemistry of nucleic acids. 11th Edition, Chepman and Hill publishers. Ltd. London, New York and Tokyo.

[39] Nahla, S.S. and Abdel-Nabi, I.M. (2006) Hypoglycemic effect of Cleome droserifolia ethanolic leaf extract in experimental diabetes and on non-enzymatic antioxidant, glycogen, thyroid hormone and insulin levels. Diabetologia Croatica, 35, 15-22.

[40] Al-Gaby, A.M.A. (1998) Amino acid compostion and biological effects of supplementing broad bean and corn protein. Nahrung, 42, 290-294. doi:10.1002/(SICI)1521-3803(199810)42:05<290::AIDFOOD290>3.0.CO;2-Y 